\title{
21-CM LINE ABSORPTION STUDY OF EXTENDED SUPERNOVA REMNANTS
}

\author{
FUMIO SATO \\ Chiba Prefecture Education Center, Chiba, Japan
}

\begin{abstract}
By using the Maryland-Green Bank Galactic 21-cm Line Survey, a series of equal-velocity contour diagrams has been constructed for the regions of the extended sources W51 and W44. The nonthermal component of W51 has no absorption features above $53 \mathrm{~km} \mathrm{~s}^{-1}$, but its thermal components have clear absorption features up to much higher velocities. From this fact, the association of the thermal and nonthermal components of W51 is rejected. Absorption features toward W44 cannot be seen above $50 \mathrm{~km}$ $\mathrm{s}^{-1}$. Kinematic distances to the nonthermal component of W51 and to W44 are $4.4 \mathrm{kpc}$ and $3.5 \mathrm{kpc}$, respectively. From peculiar brightness distributions in the equal-velocity contour diagrams I conclude that a dense $\mathrm{HI}$ cloud, as well as clouds of $\mathrm{OH}$ and $\mathrm{H}_{2} \mathrm{CO}$, is associated with W44.
\end{abstract}

Many studies of the neutral hydrogen $21-\mathrm{cm}$ line in absorption have been using profiles in the special directions of continuum sources (e.g., Kerr and Knapp, 1970; Radhakrishnan et al., 1972). They are based on the emission profiles expected in the absence of the sources. But high-resolution observations reveal many small irregularities in the brightness distribution, and it is hardly possible to get plausible expected profiles, with a few exceptions such as pulsars and some strong nonthermal sources. In the cases of extended sources and those with low surface brightness, the expected profile method is particularly ineffective. In order to inspect the absorption features on such sources, the use of an overall view of the sources and their surroundings in the twodimensional equal-velocity contour diagrams is more effective (Sato, 1968, 1969, 1970, 1973). The high-resolution survey made with the NRAO 300-ft telescope at Green Bank (Westerhout, 1969) is available for the construction of equal-velocity contour diagrams in the regions of the extended sources W51 and W44.

The source W51 consists of several thermal components and an extended nonthermal one. The 21-cm line in absorption due to the thermal components has been studied by Kerr and Knapp (1970) and Radhakrishnan et al. (1972). Kinematic distances to the thermal components were estimated from a hydrogen recombination line (Wilson et al., 1970). But no 21-cm line work on the nonthermal component has been done. Some of the constructed equal-velocity contour diagrams for radial velocities of $53 \mathrm{~km} \mathrm{~s}^{-1}$ and lower are very similar to each other and to the map of the continuum intensity. All the components of W51 show clear absorption features at those radial velocities. At higher velocities, however, no absorption feature due to the nonthermal component is seen, while those due to the thermal components are clearly seen. Thus, association of the thermal and nonthermal components is definitely rejected. The distance to the nonthermal component is $4.4 \mathrm{kpc}$ from the Sun, assuming the Schmidt (1965) model for the velocity-distance relation.

The source W44 is a well-known supernova remnant. Some authors (Goss, 1968; Goss et al., 1971; Whiteoak and Gardner, 1972) observed the $\mathrm{OH} 1720-\mathrm{MHz}$ line in emission and $\mathrm{OH}$ and $\mathrm{H}_{2} \mathrm{CO}$ lines in absorption near the edge of the shell at radial 
velocities of about $42 \mathrm{~km} \mathrm{~s}^{-1}$. Goss et al. (1971) and Whiteoak and Gardner (1972) suggested that the dense clouds of $\mathrm{OH}$ and $\mathrm{H}_{2} \mathrm{CO}$ are physically associated with the source.

In equal-velocity contour diagrams of the $21-\mathrm{cm}$ line brightness temperature, clear absorption features are seen at $V=10$ to $20 \mathrm{~km} \mathrm{~s}^{-1}$ and $V=28$ to $50 \mathrm{~km} \mathrm{~s}^{-1}$, but no trace of absorption features is seen above $V=50 \mathrm{~km} \mathrm{~s}^{-1}$. From this fact, W44 is located at a distance of $3.5 \mathrm{kpc}$ from the Sun, assuming the Schmidt (1965) model for the velocity-distance relation.

To examine whether a dense $\mathrm{H}$ I cloud is associated with any part of the source, expected profiles obtained by interpolation of the absorption dip on the constantdeclination scans or by averaging the intensities at several points outside the source are not sufficiently reliable. Therefore, I have constructed expected equal-velocity contour diagrams by using the 1414-MHz continuum survey (Altenhoff et al., 1970) made with the NRAO 300-ft telescope. I add a constant multiple of the continuum brightness to the observed $21-\mathrm{cm}$ brightness, which will give the brightness distribution with the source subtracted, assuming constant optical depth. Figure 1 is such an expected equal-velocity contour diagram for $V=20 \mathrm{~km} \mathrm{~s}^{-1}$, assuming a constant optical depth of 0.29 over the source W44. No feature associated with the source is

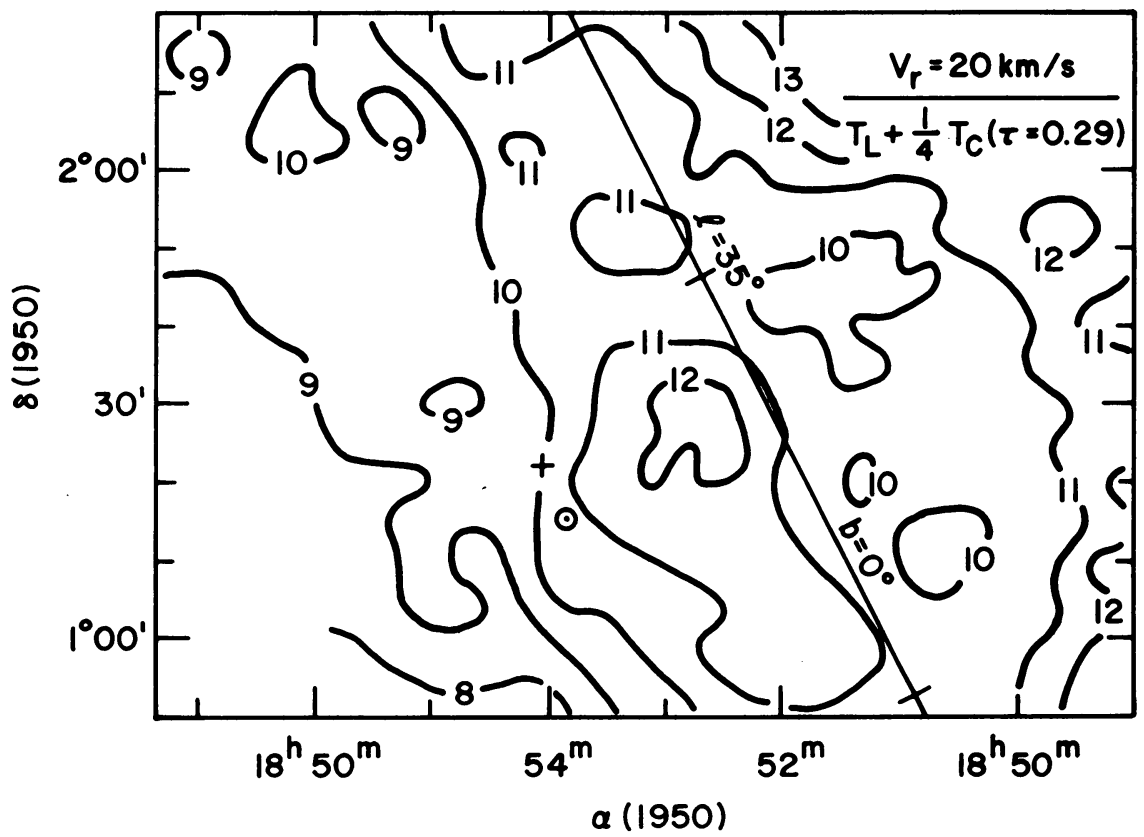

Fig. 1. Equal-velocity contour diagram at $V=20 \mathrm{~km} \mathrm{~s}^{-1}$ assuming a constant optical depth of 0.29 over the source W44, derived from the 'Maryland-Green Bank Galactic 21-cm Line Survey', 2nd edn. (Westerhout, 1969) and the 1414-MHz map observed with the NRAO 300-ft telescope (Altenhoff et al., 1970). Contour unit is $5 \mathrm{~K}$ in $T_{\mathrm{a}}$. A circled dot shows the position of the continuum maximum, and a cross shows that of the maximum opacity of the $\mathrm{OH} 1667-\mathrm{MHz}$ line (Goss et al., 1971). 


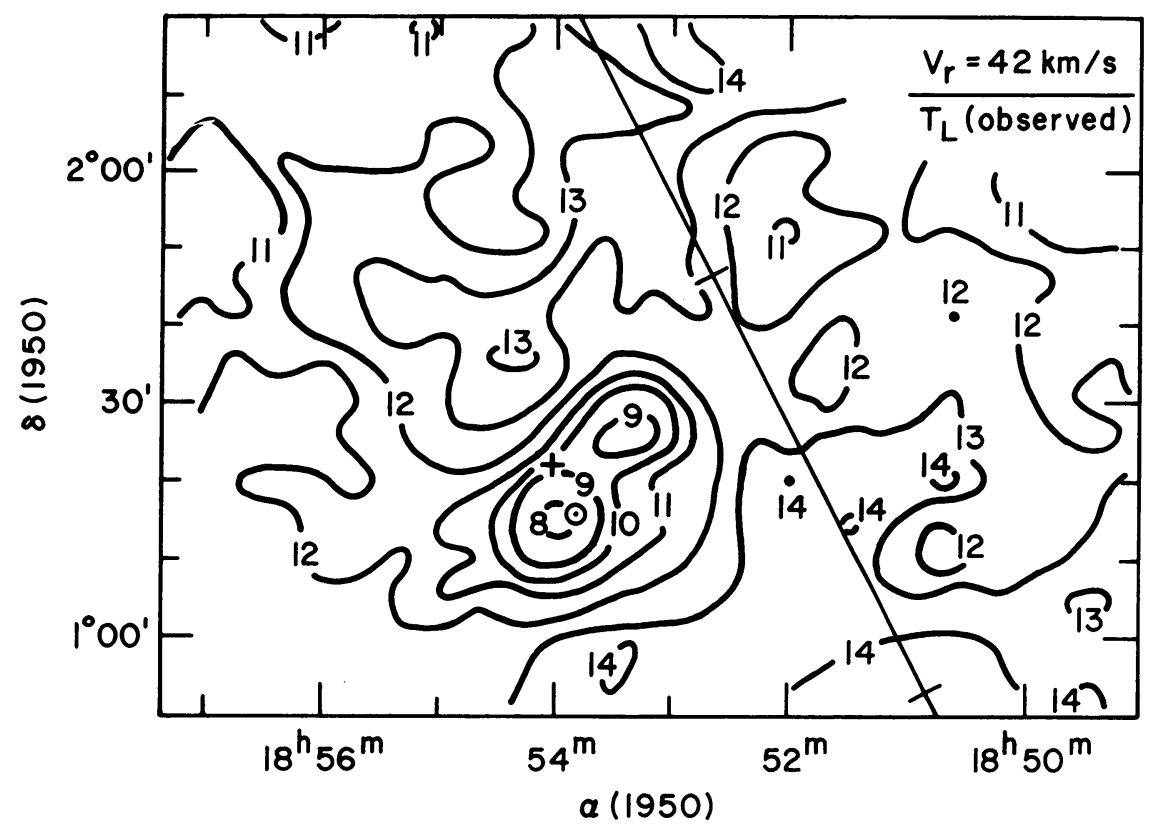

Fig. 2. Equal-velocity contour diagram for a region of $\mathrm{W} 44$ at $V=42 \mathrm{~km} \mathrm{~s}^{-1}$ derived from the 'MarylandGreen Bank Galactic 21-cm Line Survey', 2nd edn. (Westerhout, 1969). See Figure 1.

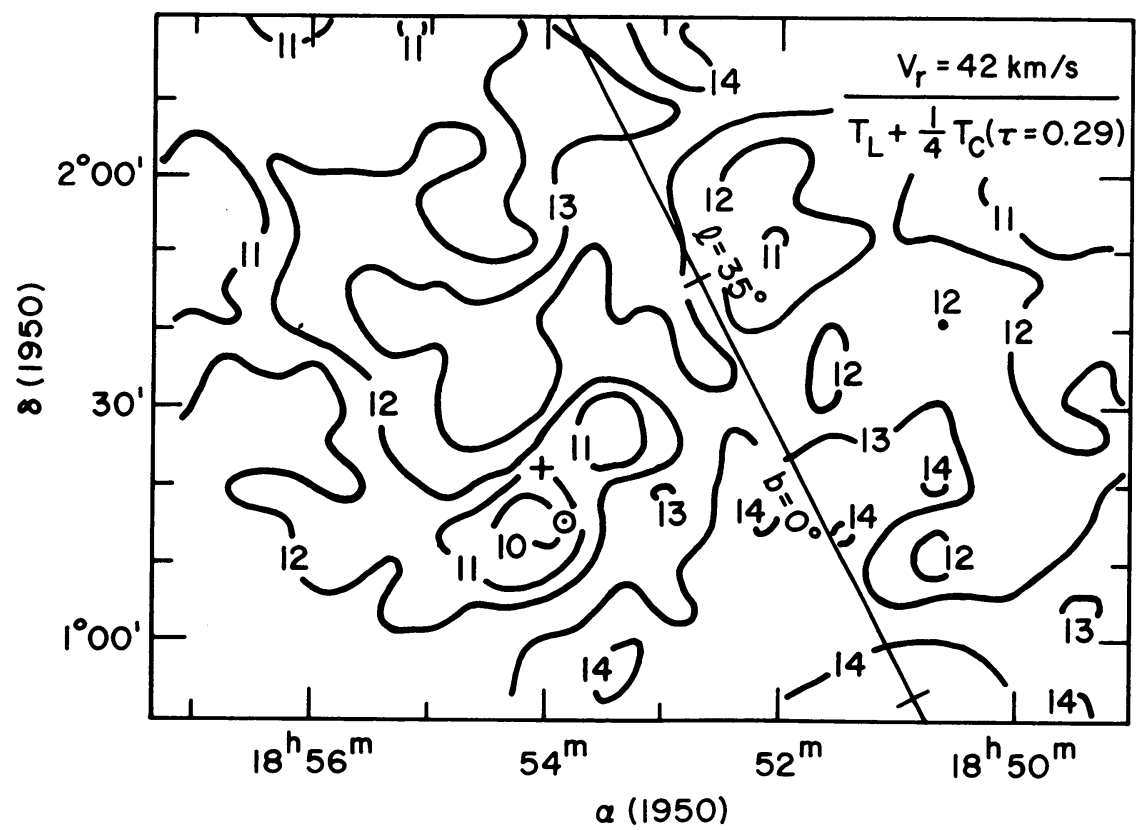

Fig. 3. Equal-velocity contour diagram at $V=42 \mathrm{~km} \mathrm{~s}^{-1}$ assuming a constant optical depth of 0.29 over W44. See Figure 1. 


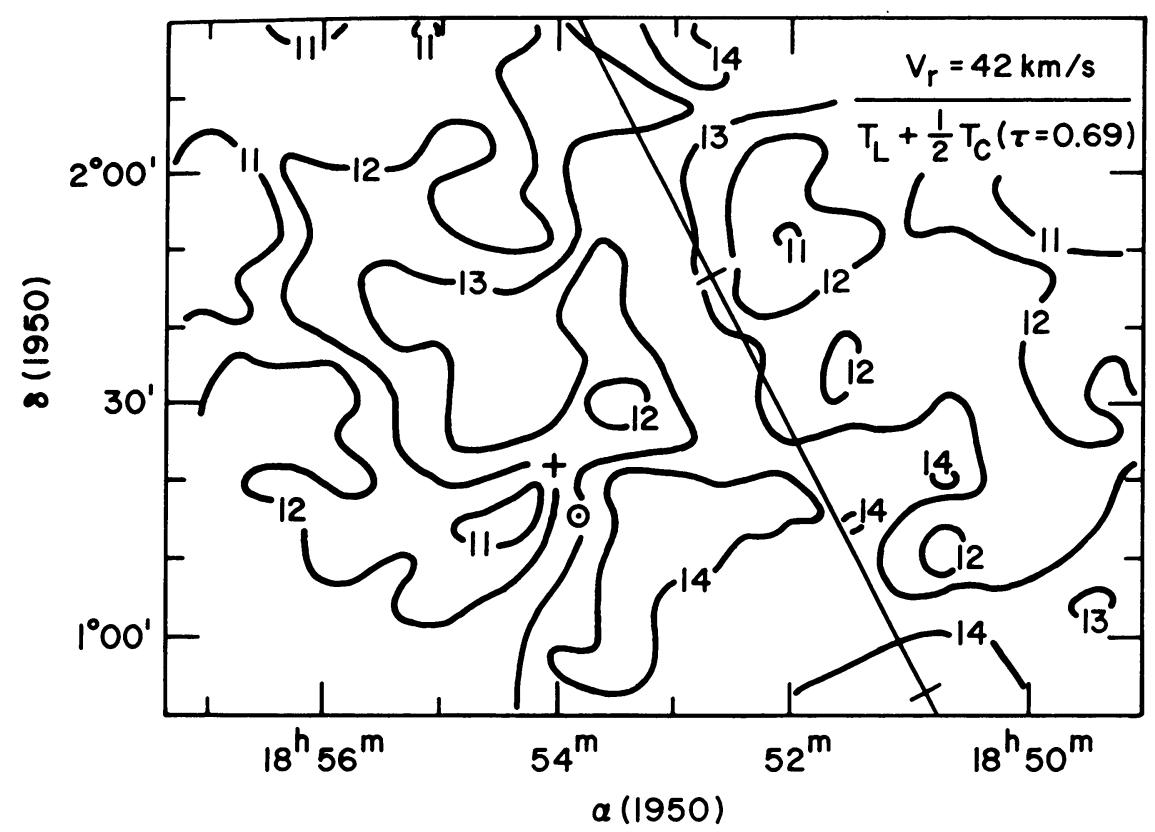

Fig. 4. Equal-velocity contour diagram at $V=42 \mathrm{~km} \mathrm{~s}^{-1}$ assuming a constant optical depth of 0.69 over W44. See Figure 1.

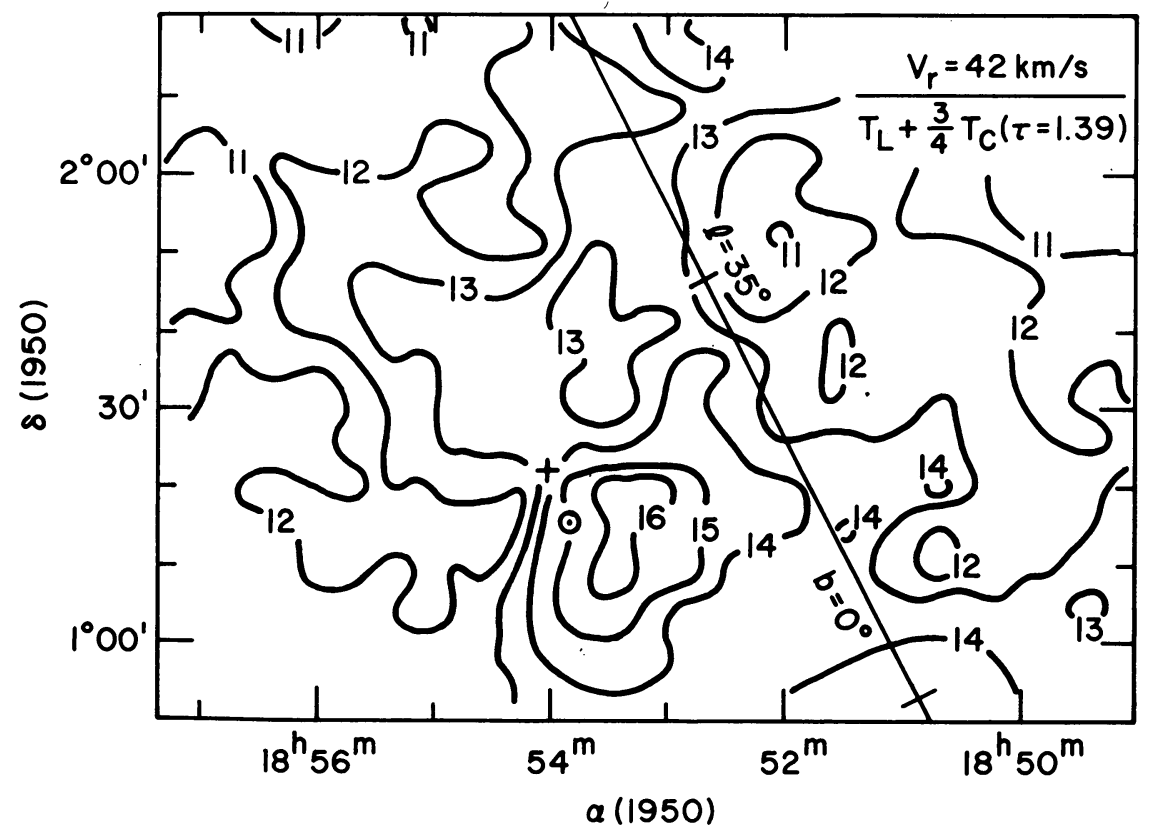

Fig. 5. Equal-velocity contour diagram at $V=42 \mathrm{~km} \mathrm{~s}^{-1}$ assuming a constant optical depth of 1.39 over W44. See Figure 1. 
seen, and the smooth distribution of the brightness shows that the assumption of constant optical depth is reasonable.

Figure 3 is an expected equal-velocity contour diagram for $V=42 \mathrm{~km} \mathrm{~s}^{-1}$, assuming a constant optical depth of 0.29 . It resembles well the observed intensity contour diagram in Figure 2 and still shows a rather clear absorption feature, but its bottom is displaced to the northeast of the source from the vicinity of the continuum peak. This phenomenon is more clearly seen in Figure 4, which is for the same velocity assuming a constant optical depth of 0.69 over the source. The southwestern part of the source begins to emerge, but in its northeastern part there can be seen a trough along the shell through the region of large optical depth of the absorbing $\mathrm{OH}$ and $\mathrm{H}_{2} \mathrm{CO}$. In Figure 5 for $V=42 \mathrm{~km} \mathrm{~s}^{-1}$, assuming a constant optical depth of 1.39, the continuum emission of the source added to the observed $21-\mathrm{cm}$ line intensity is quite strong, and the western half of the source appears. In the eastern half, however, the trough is barely filled, and there is no enhancement of emission due to the added continuum radiation of the source. These facts imply that at $V=42 \mathrm{~km} \mathrm{~s}^{-1}$, $\mathrm{H} \mathrm{I}$ gas in the southwestern part of W44 has a small optical depth of 0.29 or so, but in the northeastern part its optical depth is as large as 1.39 or so. Such features are seen also in the contour diagram for the velocity range from 40 to $46 \mathrm{~km} \mathrm{~s}^{-1}$. The dense $\mathrm{H} \mathrm{I}$ gas agrees well in position and velocity with $\mathrm{OH}$ and $\mathrm{H}_{2} \mathrm{CO}$ concentrations which have been suggested to be associated with the source (Goss et al., 1971; Whiteoak and Gardner, 1972). Thus, I have come to the conclusion that a dense H I gas cloud with a radial velocity of 40 to $46 \mathrm{~km} \mathrm{~s}^{-1}$ is associated with the shell of the source W44.

\section{References}

Altenhoff, W. J., Downes, D., Goad, L., Maxwell, A., and Rinehart, R.: 1970, Astron. Astrophys. Suppl. 1, 319.

Goss, W. M.: 1968, Astrophys. J. Suppl. 15, 131.

Goss, W. M., Caswell, J. L., and Robinson, B. J.: 1971, Astron. Astrophys. 14, 481.

Kerr, F. J. and Knapp, G. R.: 1970, Australian J. Phys. Astrophys. Suppl., No. 18, 9.

Radhakrishnan, V., Goss, W. M., Murray, J. D., and Brooks, J. W.: 1972, Astrophys. J. Suppl. $24,49$.

Sato, F.: 1968, Publ. Astron. Soc. Japan 20, 303.

Sato, F.: 1969, Ann. Tokyo Astron. Obs. Second Ser. 11, 67.

Sato, F.: 1970, Ann. Tokyo Astron. Obs. Second Ser. 12, 1.

Sato, F.: 1973, Publ. Astron. Soc. Japan 25, 135.

Schmidt, M.: 1965, in A. Blaauw and M. Schmidt (eds.), Galactic Structure, University of Chicago Press, Chicago, p. 513.

Westerhout, G.: 1969, 'Maryland-Green Bank Galactic 21-cm Line Survey', 2nd edn., University of Maryland, College Park, Maryland.

Whiteoak, J. B. and Gardner, F. F.: 1972, Astron. Astrophys. 21, 159.

Wilson, T. L., Mezger, P. G., Gardner, F. F., and Milne, D. K.: 1970, Astrophys. Letters 5, 99.

Fumio Sato

Chiba Prefecture Education Center, 2-10-1 Katsuragi, Chiba, Japan 


\section{DISCUSSION}

$J$. R. Dickel: Maps of the $21-\mathrm{cm}$ line at various radial velocities in the vicinity of IC 443 show a hole right at the position of the source at all velocities, which suggests that the SNR is at or behind all the significant hydrogen in that direction. Depending on how one continues the Perseus arm around to that area through the anticenter of the Galaxy, we can obtain various estimates of the distance, but the closest values are about $2.2 \mathrm{kpc}$ (using Roberts' shock model). This would put the distance well beyond that of the Hil region to the northeast of IC 443, which several people have suggested is responsible for the retardation of the shell's expansion. (The distance estimate of $550 \mathrm{pc}$ is by Racine from photometry of a B8 star which illuminates the reflection nebula.) At positive velocities the neutral hydrogen appears to be part of the general galactic emission, but at $V=-6$ to $-10 \mathrm{~km} \mathrm{~s}^{-1}$ there is a definite cloud to the northeast of IC 443 , and it is probably this which retards the expansion and may make it old enough to match the pulsar age.

Guélin: Minkowski has derived a distance of $\gtrsim 2 \mathrm{kpc}$ for IC 443 from the motion of the filaments. (IAU Symp. 9 (1959), 315.)

Mills: The distance of IC443 on the $\Sigma-D$ relation I presented also is about $2-5 \mathrm{kpc}$. It would be hard to accept $550 \mathrm{pc}$.

Caswell: How do you determine a kinematic distance for the neutral hydrogen in the direction of IC 443, near the anticenter?

Dickel: From the shock model by Roberts (Astrophys. J. 173 (1972), 259), who continued the Perseus arm around to this direction. 